\title{
Characteristics of Child and Adolescent Psychiatry Consultations at a University Hospital and Accuracy Rates of Recognition of Childhood Psychiatric Diseases by Nonpsychiatry Specialists
}

\author{
Zehra Topal $^{1(\mathbb{D})}$, Mehmet Karadag ${ }^{1(\mathbb{I D})}$, Baran Calisgan ${ }^{1(\mathbb{D})}$, Fatma Subaşi Turgut ${ }^{1(\mathbb{D})}$, \\ Cem Gokcen ${ }^{1(\mathrm{ID})}$, Ilhan Bahsi ${ }^{2(\mathrm{ID})}$ \\ ${ }^{1}$ Department of Child and Adolescent Psychiatry, Faculty of Medicine, Gaziantep University, Gaziantep, Turkey \\ ${ }^{2}$ Department of Anatomy, Faculty of Medicine, Gaziantep University, Gaziantep, Turkey
}

Copyright@Author(s) - Available online at https://dergipark.org.tr/en/pub/mbsjohs

Content of this journal is licensed under a Creative Commons Attribution-NonCommercial 4.0 International

License,

Received: 16 February 2021, Accepted: 29 March 2021, Published online: 30 April 2021

(C) Ordu University Institute of Health Sciences, Turkey, 2021

\begin{abstract}
Objective: Physical and mental health are closely related. Psychiatric problems increase in the presence of a physical illness. Consultation liaison psychiatry provides a combination of medical treatment, psychiatric treatment, and psychosocial care to patients when necessary. In this study, we aimed to examine the consultation-liaison services provided by the child and adolescent psychiatry department of a university hospital within two years and to examine the accuracy rates of recognition of childhood psychiatric diseases nonpsychiatry specialists.

Methods: Consultations for pediatric patients from other clinics were screened retrospectively at Gaziantep University Hospital between January 1st, 2018, and December 31st, 2019. Data relating to the departments requesting a consultation, reasons for consultation, diagnoses made by children and adolescent psychiatrists, and psychiatric treatments were evaluated.

Results: A child and adolescent psychiatry consultations were requested for 290 children over a two-year period. The average age of the cases for whom consultation was requested was 12.1 , and $61 \%$ of the cases were female. The most common reason for consultation was suicide attempts (26.6\%). The most common mental disorder was depression (19\%), while the most common intervention was psychoeducation (48.9\%). The disorders with the highest diagnostic accuracy were delirium (100\%), autism (100\%), substance use disorders $(80 \%)$, and mental disability $(70 \%)$, while the disorders with the lowest diagnostic accuracy were psychotic disorder $(0 \%)$, depression $(\% 18,2)$ and anxiety disorder $(22.2 \%)$.

Conclusion: Mental disorders are common in children with physical illnesses, but the rate of correct recognition of childhood mental disorders by other physicians is low. The high rates of psychiatric disorders highlight the importance of educating physicians that work with children about childhood mental problems and cooperation between child psychiatrists and other physicians.

Key words: Psychiatry, Child, Consultation

Suggested Citation Topal Z, Karadag M, Calisgan B, Subasi Turgut F, Gokcen C, Bahsi I. characteristics of child and adolescent psychiatry consultations at a university hospital and accuracy rates of recognition of childhood psychiatric diseases by nonpsychiatry specialists. Mid Blac Sea Journal of Health Sci, 2021; 7(1):88-96
\end{abstract}

Address for correspondence/reprints:

Telephone number: +90 (342) $3606060 / 76468$

Zehra Topal

E-mail: zehratopal86@gmail.com 


\section{Introduction}

Until recently, the general medical approach focused on the biomedical model in which physical health was prioritized, whereas the currently accepted approach gives weight to the biopsychosocial model that treats the person as a whole in terms of physical, spiritual and social aspects (1). As a holistic approach, the biopsychosocial model emphasizes that all biological, psychological, environmental and sociocultural factors should be taken into account while treating the patient. Along the same lines, World Health Organization (WHO) defines the state of being healthy as "a state of complete physical, mental and social well-being", which is a definition compatible with the biopsychosocial model (2).

Consultation-liaison psychiatry (CLP) is a branch of psychiatry that is based on the biopsychosocial model, which concerns with the emotional and behavioral assessment and treatment of patients referred by non-psychiatric physicians, providing psychiatric treatment and psychosocial care simultaneously with the medical treatment to patients (3). Previously conducted studies established that the hospitalization rates were higher in cases with both mental health disorders and physical illnesses compared to the cases with either physical illness or mental illness (4). Similarly, many studies have reported that the prognosis was worsened, the duration of hospital stay was prolonged, and the cost of the disease to the healthcare system was higher in cases with a psychiatric disorder as comorbidity (58). Additionally, psychiatric consultation has been shown to improve outcomes in inpatients $(9,10)$.

Childhood mental health problems are increasing gradually. A national survey study recently conducted in U.S. revealed that approximately $17 \%$ of children have a mental health problem (11), whereas a study conducted nationwide in Turkey reported that $37.6 \%$ of children have mental health problems and that $17 \%$ of children have a psychopathology impairing their functionality (12). It is a known fact that the incidence of psychopathology is higher in children and adolescents with medical diseases (13). Despite the high prevalence of psychiatric diseases in childhood, the high rates of psychiatric comorbidities in physical diseases, and the fact that psychiatric diseases increase mortality and morbidity, psychiatric diseases are still not recognized and treated sufficiently (14).

Many studies have been conducted in Turkey investigating the psychiatric consultations, yet the number of studies investigating the rates of accurate prediagnoses made for the psychiatric diseases by non-psychiatric physicians is quite limited (15-19). A thorough review of the literature revealed only a few studies conducted on the rates of accurate recognition of adult psychiatric diseases (20-22). Still, it did not reveal even one study conducted on the rates of accurate recognition of child and adolescent psychiatric diseases.

In view of the foregoing, in this study, it was aimed to investigate the psychiatric consultation services provided by Gaziantep University Faculty of Medicine Department of Child and Adolescent Psychiatry in the last two years and the rates of accurate recognition of child psychiatric diseases by non-psychiatric physicians.

\section{Methods}

This study was carried out in accordance with the principles of the Declaration of Helsinki and was approved by the Gaziantep University Clinical Research Ethics Committee in advance (Date: 22.06.2020, decision number: 202).

\section{Selection and Characterization of Cases}

The patient files regarding the psychiatric consultations requested from Gaziantep University Faculty of Medicine Department of Child and Adolescent Psychiatry between January 1st, 2018 and December 31st, 2019, were reviewed retrospectively. Those with missing information in the file records were excluded from the study. All consultations requested for children aged 0-18 were included in the study. Diagnostic assessments were made based on the evaluation of the interviews conducted with children and adolescents for whom psychiatric consultations were requested, family members of these children and adolescents, and the healthcare professionals working in the department that requested the psychiatric consultations, in accordance with the diagnostic criteria set forth in the Diagnostic and Statistical Manual of Mental Disorders, Fifth Edition (DSM-5).

\section{Statistical analysis}

The data obtained were evaluated statistically. Descriptive statistics of the obtained data were made. SPSS 22.0 package program was used for analysis (IBM Corporation; Armonk, NY, USA), and $\mathrm{P}<0.05$ was considered significant. The sociodemographic characteristics of the children and adolescents, the departments which requested the psychiatric consultations, the reasons for requesting the psychiatric consultations, the psychiatric diagnoses made as a result of the consultations, and the interventions performed by the child psychiatrist were analyzed by descriptive statistics (median, standart deviation, frequency distribution). 
Diagnostic accuracy in psychiatric consultations was calculated by percantages of the diagnoses confirmed by the psychiatrist to all consultations requested for that diagnosis.

\section{Results}

It was determined that a child psychiatry consultation was requested from various departments for a total of 290 children and adolescents between January 1st, 2018 and December 31st, 2019. During this period, the number of children aged 0-18 who applied to the clinic subject to this study was 134,030 in total, and child psychiatry consultations were requested for $0.002 \%$ of these patients. The number of patients that received inpatient treatment was 10,491 and child psychiatry consultations were requested for $0.01 \%$ of these patients, whereas child psychiatry consultations were requested for $0.001 \%$ of the patients that have been receiving outpatient treatment. The mean age of the children for whom psychiatric consultations were requested was calculated as $12.1 \pm 3.8$ ( 2 to 18$)$. One hundred seventy-seven of these cases were female $(61.03 \%)$, and $113(38.97 \%)$ of them were male. $73.8 \%(\mathrm{n}=$ 214) of the children and adolescents who were provided psychiatric consultation services were found to have previously applied to a child psychiatry outpatient clinic, whereas the remaining $26.2 \%$ ( $n=$ 76) of the children and adolescents were determined to have received psychiatric consultation services for the first time.

The clinic which requested the highest rate (43.7\%) of psychiatric consultations was the pediatric inpatient service, followed by the emergency service, pediatric outpatient clinic, and intensive care anesthesia service in descending order (Table-1).
The most common reason $(\mathrm{n}=77)$ for requesting psychiatric consultations was suicide attempt, followed by agitation, depressive symptoms, noncompliance to treatment, anxiety symptoms, somatic complaints, psychotic symptoms, sleep and eating problems, in descending order (Table-2).

The most common diagnoses made following the assessment of the CLP team was depression (21.9\%), followed by adjustment disorder (15.8\%), mental disability (14.3\%), anxiety disorders $(11.2 \%)$ and attention deficit hyperactivity disorder $(7.7 \%)$, in descending order (Table-3).

There was no prediagnosis in the consultation notes of $70 \%$ of the patients. The most common prediagnoses indicated in the consultation notes that include prediagnoses were depression (7.6\%), anxiety disorder (5.9\%), psychosis (4.5\%), and mental disability (3.4\%), respectively. Comparison of the prediagnoses with the final diagnoses made based on the psychiatric assessment revealed that autism, delirium, substance use disorders and mental disability were the disorders with the highest rates of accurate prediagnoses as compared to the final diagnoses, whereas that psychosis, depression, and anxiety were the disorders with the highest rates of misdiagnoses made during the prediagnosis stage as compared to the final diagnoses (Table-4).

In terms of psychiatric interventions, following up of the patients by providing them psychoeducation was determined to be the most common (48.9\%) form of intervention. Other commonly used treatment options included the use of selective serotonin reuptake inhibitors (SSRI), antipsychotics, benzodiazepines, and stimulants, in descending order. No intervention was deemed necessary in $13.4 \%$ of the patients (Table-5).

Table-1: Clinics That requested psychiatric consultations

\begin{tabular}{lll}
\hline Clinic & Number* & \% \\
\hline Pediatric Inpatient Service & 127 & 43,7 \\
\hline Emergency Service & 116 & 40,0 \\
\hline Pediatric Outpatient Clinic & 39 & 13,4 \\
\hline Intensive Care Anesthesia Service & 8 & 2,7 \\
\hline
\end{tabular}

* Number of patients for whom a non-psychiatric physician requested psychiatric consultations 
Table-2: Reasons indicated for psychiatric consultation requests

\begin{tabular}{lll}
\hline Reasons & Number* & \% \\
\hline Suicide Attempt & 77 & 26,6 \\
\hline Agitation & 35 & 12,1 \\
\hline Depressive Symptoms & 22 & 7,9 \\
\hline Non-compliance to Treatment & 19 & 6,6 \\
\hline Anxiety Symptoms & 18 & 5,9 \\
\hline Somatic Complaints & 17 & 5,9 \\
\hline Psychotic Symptoms (Hallucinations / Delusions) & 15 & 5,2 \\
\hline Sleeping and Eating Problems & 10 & 3,4 \\
\hline Mental Retardation Symptoms & 10 & 3,4 \\
\hline Conversive Symptoms & 8 & 2,8 \\
\hline Side Effects Caused by Psychiatric Medications & 7 & 2,4 \\
\hline Presence of a History of Psychiatric Disorders & 5 & 1,7 \\
\hline Alcohol/Substance Use & 5 & 1,7 \\
\hline Attention Deficit Hyperactivity Disorder Symptoms & 5 & 1,7 \\
\hline Other Reasons & 37 & 12,8 \\
\hline
\end{tabular}

* Number of patients for whom a non-psychiatric physician requested psychiatric consultations

Table-3: Final diagnoses made on the basis of the requested consultations

\begin{tabular}{lll}
\hline Type of Diagnoses & Number* & \% \\
\hline Depression & 43 & 21,9 \\
\hline Adjustment Disorder & 31 & 15,8 \\
\hline Attention Deficit Hyperactivity Disorder & 15 & 7,7 \\
\hline Mental Disability & 28 & 14,3 \\
\hline Anxiety Disorder & 22 & 11,2 \\
\hline Conversion & 14 & 7,1 \\
\hline Delirium & 10 & 5,1 \\
\hline Autism Spectrum Disorder & 7 & 3,6 \\
\hline Substance Use Disorder & 6 & 3,1 \\
\hline Psychosis & 3 & 1,5 \\
\hline Bipolar Disorder & 7 & 3,6 \\
\hline Obsessive Compulsive Disorder & 5 & 2,6 \\
\hline Dystonia & 2 & 1,0 \\
\hline Eating Disorder & 3 & 1,5 \\
\hline
\end{tabular}

* Number of patients for whom a non-psychiatric physician requested psychiatric consultations

Table-4: Rates of accuracies of the prediagnoses indicated by the non-psychiatric physicians in the consultation notes

\begin{tabular}{lccc} 
& $\begin{array}{c}\text { Prediagnoses Made by the } \\
\text { Non-Psychiatric Physicians }\end{array}$ & $\begin{array}{c}\text { Prediagnoses Made by the } \\
\text { Child Psychiatrists }\end{array}$ & $\begin{array}{c}\text { Rates of the Prediagnoses } \\
\text { Confirmed }\end{array}$ \\
\hline Depression & $\mathrm{n}^{*}$ & 4 & 18,2 \\
\hline Anxiety Disorder & 22 & 4 & 22,2 \\
\hline Psychotic Disorder & 18 & 0 & 0 \\
\hline Mental Disability & 13 & 7 & 70 \\
\hline Conversion & 10 & 4 & 50 \\
\hline Substance Use Disorder & 8 & 4 & 80 \\
\hline ADHD & 5 & 2 & 40 \\
\hline Delirium & 5 & 2 & 100 \\
\hline Autism & 2 & 2 & 100
\end{tabular}

ADHD: Attention Deficit Hyperactivity Disorder

*Number of patients for whom non-psychiatric physicians made prediagnoses

**Number of patients, whose prediagnoses were confirmed by the child psychiatrists 
Table-5. Interventions performed by the child and adolescent psychiatrists

\begin{tabular}{lll}
\hline Types of Interventions/Medications & Number* & \% \\
\hline Patients for whom no intervention was deemed necessary & 38 & 13,4 \\
\hline Psychoeducation and follow-up & 139 & 48,9 \\
\hline SSRIs & 48 & 16,9 \\
\hline Antipsychotics & 44 & 15,5 \\
\hline Benzodiazepines & 9 & 3,2 \\
\hline Methylphenidate & 6 & 2,1 \\
\hline
\end{tabular}

SSRI: Selective Serotonin Reuptake İnhibitors

* Number of patients for whom psychiatric consultations were requested by a non-psychiatric physician

\section{Discussion}

Despite the fact that the evidence indicating the importance of CLP is growing, it is noteworthy that the rates of patients for whom child psychiatry consultations were requested have been quite low in Turkey, as it can be seen from the low rates of patients that have been receiving inpatient treatment and for whom child psychiatry consultations were requested, which were reported to be between $1.2 \%$ and $2.3 \%$ $(16-18,23)$. In comparison, in this study, it was found that child and adolescent psychiatry consultations were requested only for $0.01 \%$ of the children that have been receiving inpatient treatment and $0.001 \%$ of the children that have been receiving outpatient treatment. These rates were lower than the respective rates reported in the above-mentioned studies conducted in Turkey, and substantially lower than the rates of patients that have been receiving inpatient treatment abroad and for whom child psychiatry consultations were requested, which were reported to be between $27 \%$ and $66 \%(24,25)$. It has been suggested that the low rate of child psychiatry consultations in Turkey may be due to several factors such as physicians' difficulties in recognizing mental symptoms, the fact that the physicians do not have enough knowledge about the effect of psychiatric comorbidities on medical pathology, the fact that the physicians do not think that psychiatric consultations will be beneficial, non-existence of effective pediatric CLP teams in hospitals and absence of effective communication and collaborative approach between the CLP teams and other physicians $(15,16)$.

Organization of awareness-raising trainings by CLP teams for non-psychiatric physicians and holding meetings where complex cases are discussed with a multidisciplinary perspective by psychiatrists and non-psychiatric physicians in psychiatry clinics can raise awareness about the issue and contribute to the more effective use of CLP services. As a matter of fact, the results of relevant studies available in the literature indicate that cooperation among physicians and consultations have increased as a result of the development and implementation of new models that provide effective use of CLP $(26,27)$.

In this study, only patients for whom the child and adolescent psychiatric consultations were requested in writing could be evaluated. It is also known that there are cases frequently encountered in outpatient clinics where a psychiatric consultation is not officially requested yet the patients are verbally recommended to apply to the psychiatry/child psychiatry clinic, especially in cases when the complaints of the patients cannot be explained by a medical condition. Taking into consideration the prejudice against the psychiatric specializations in the Turkish society, it would not be inaccurate to make a prediction that some of the patients would leave the health centers without applying to psychiatry clinics after such recommendations communicated verbally, which might be one of the reasons for the low rate of psychiatric consultation requests found in this study as well as in other studies conducted in Turkey. For this reason, it is essential to seek official consultations rather than making verbal recommendations to that effect, in order to ensure that the patients are assessed psychiatrically and receive the treatments they need.

In this study, the mean age of the children and adolescents for whom a psychiatric consultation was requested was calculated as 12 . The rate of psychiatric consultations requested for girls $(61.03 \%)$ was significantly higher than the rate of psychiatric consultations requested for boys, which is a compatible finding with the results reported in the literature, considering that the reported rates of psychiatric consultations requested for girls' range between $54 \%$ and $70 \%(18,23,28-32)$. It is known that psychiatric referrals are more common in boys in the pre-adolescence period, however that the prevalence of psychopathologies increases in girls as opposed to boys beginning with early adolescence 
(33). It has been suggested that the strengthening in response to stress with the effect of cortisol and estrogen the levels of which increase with puberty, makes adolescent girls more sensitive to psychological stress and internalizing disorders (34). Thus, the fact that psychiatric consultations are requested more for adolescent girls compared to the adolescent boys may be attributed to the increase in the psychopathology observed in girls during the adolescence period.

In this study, the most common reason for requesting psychiatric consultations was found to be suicide attempt, followed by agitation and depressive symptoms. Similarly, a review of the national literature revealed that the most common reasons submitted for requesting psychiatric consultations were suicidal attempts $(16,31,35)$ and depressive symptoms $(15,16,18)$. On the other hand, it was reported in several studies that the most common reasons submitted for requesting psychiatric consultations internationally were behavioral problems and hyperactivity $(25,36)$. In a survey study including 64 independent pediatric CLP services, Shaw et al. (37) reported that the most common reasons for requesting psychiatric consultations were suicide risk assessment, presence of medically unexplained symptoms, compliance problems with medical illness, assessment in terms of psychopharmacological treatment, delirium and noncompliance to treatment.

In sum, suicide attempts constitute one of the main reasons for requesting a child psychiatry consultation in both domestic and international studies. Adolescents often attempt to commit suicide compulsively rather than as a result of a planned attempt $(17,31)$. Adolescence is a period where biological, spiritual and social growth accelerate. The biological, psychological and social changes that occur can be emotionally challenging for adolescents, and it can be difficult for them to cope with these emotional difficulties since their experience and maturation are not sufficient just yet. Consequently, adolescents may compulsively resort to suicide in such moments which they have difficulty coping. For this reason, it is very important to perform a psychiatric assessment of every young person presenting with acute intoxication in terms of a possible suicide attempt.

Comparison of the prediagnoses with the final diagnoses made based on the psychiatric assessment revealed that the most common prediagnoses made by $\mathrm{t}$ non-psychiatric physicians were depression and anxiety disorder. However, it was found that most of these prediagnoses were not confirmed by the child psychiatrists. The psychiatric disorders which were misdiagnosed the most by non-psychiatric physicians as compared to the final diagnoses made by based on the psychiatric assessment were found to be the psychotic disorders; whereas prediagnoses made in respect of autism, delirium, substance use disorders and mental disability were determined to be the disorders which were accurately diagnosed the most as compared to the final diagnoses made by based on the psychiatric assessment. Nevertheless, the relatively low number of prediagnoses of delirium and autism, each of which were diagnosed in 2 children and adolescents, compared to the total number of final diagnoses of delirium and autism, that is, 10 children and adolescents with delirium and 7 children and adolescents with autism, which also includes the number of children and adolescents diagnosed with delirium and autism however not referred by a non-psychiatric physician for consultation, has been interpreted such that most of these disorders were not known to the physicians that have requested psychiatric consultations.

Several studies conducted in the adult population have demonstrated that the non-psychiatric physicians predominantly recognize cognitive disorders, delirium and substance use disorders, but recognize depression, anxiety and psychotic disorders only occasionally $(20,38,39)$. In a study conducted in an adult population in Turkey, it was stated that the prediagnoses in respect of the respective mental disorder were not made in more than half of the patients for whom a psychiatric consultation was requested due to symptoms such as agitation, depressive findings and symptoms, anxiety and conversion symptoms, despite the fact that it would be expected that the diagnoses in question were accurately made given that the stated symptoms and findings clearly indicated the respective mental disorder (40). The respective results obtained in this study in respect of the pediatric age group were found to be comparable to the above-mentioned results reported in respect of the adult population.

In terms of psychiatric interventions, the most common form of intervention performed by the CLP team subject to this study was found to be patient follow-ups through psychoeducation. Psychoeducation includes educational interventions intended to teach the patients with mental or physical illnesses about their illnesses and their emotional responses to the disease, to improve their ability to cope with difficult situations, to increase their adaptation skills, and to ensure their compliance with the treatment (41-42). The aim of these interventions is to teach the patients how to cope with their 
problems, to help them understand the situation they are in, and to increase their general well-being and quality of life, through furnishing them with the necessary knowledge and ensuring them to adopt the appropriate behavioral changes. The effectiveness of psychoeducation when used alone as an intervention method has been shown based on evidence, yet it can also be used in combination with other treatment methods (42).

Second to the psychoeducation, the next most common form of intervention performed by the CLP team subject to this study was found to be pharmacotherapy, and the most commonly pharmacological agents used in this regard were SSRIs, antipsychotics, benzodiazepines and methylphenidate, respectively. A review of the literature in this respect revealed that pharmacotherapy was used as a method of intervention by the CLP teams in $28 \%$ to $85 \%$ of the patients, for whom a psychiatric consultation was requested $(15-18,28)$. In this study, it was determined that pharmacotherapy was used as a method of intervention by the CLP team in $37.7 \%$ of the patients, for whom a psychiatric consultation was requested, and psychoeducation practices were found to be ahead of medical treatment. Psychopharmacological approaches are preferred by physicians over psychotherapeutic approaches, which are difficult to apply due to reasons such as time limitation and the need for specially trained personnel in children and adolescents with physical diseases (43). The fact that the clinic subject to this study is a child-adolescent psychiatry clinic that focuses on psychotherapy training and practices might have led the respective CLP team to attach priority to psychosocial interventions.

\section{Conclusions}

In conclusion, the findings of this study indicate that most of the children with physical illnesses and for whom psychiatric consultation was requested had a mental disorder. However, the rate of requesting child and adolescent psychiatric consultations is very low. Child mental health diseases are not recognized correctly by non-psychiatric physicians. Autism, delirium, substance use disorders and mental disability were found to be the disorders with the highest rates of accurate prediagnoses as compared to the final diagnoses, whereas psychosis, depression, and anxiety were found to be the disorders with the highest rates of misdiagnoses made during the prediagnosis stage as compared to the final diagnoses. Nevertheless, the relatively low number of prediagnoses of delirium and autism, compared to the total number of final diagnoses of delirium and autism, which also includes the number of children and adolescents diagnosed with delirium and autism however not referred by a non-psychiatric physician for consultation, has been interpreted such that most of these disorders were not known to the physicians that have requested psychiatric consultations, suggesting that it is highly likely that most of the children with delirium and autism have been overlooked.

Ethics Committee Approval: Study protocol was approved by the Gaziantep University Clinical Research Ethics Committee in advance (Date: 22.06.2020, decision number: 202).

Peer-review: Externally peer-reviewed.

\section{Author Contributions:}

Concept: Z.T, M. K, B.C, F.S.T, C.G, I.B, Design: Z.T, M. K, B.C, F.S.T, C.G, I.B Literature Search: Z.T, M. K, B.C, F.S.T, C.G, I.B, Data Collection and/or Processing: Z.T, M. K, B.C, F.S.T, C.G, I.B, Analysis and/or Interpretation: Z.T, M. K, B.C, F.S.T, C.G, I.B, Writing: Z.T, M. K, B.C, F.S.T, C.G, I.B.

Conflict of Interest: No conflict of interest was declared by the author.

Financial Disclosure: The author declared that this study hasn't received no financial support.

\section{References}

1. Farre A, Rapley T. The New Old (and Old New) Medical Model: Four Decades Navigating the Biomedical and Psychosocial Understandings of Health and Illness. Healthcare (Basel). 2017;5(4):88. doi: 10.3390/healthcare5040088.

2. Huber M, Knottnerus JA, Green L, van der Horst $\mathrm{H}$, Jadad AR, et al. How should we define health? BMJ 2011;343:d4163.

3. Leigh H, Streltzer JM. Handbook of consultationliaison psychiatry. New York, NY, US: Springer Science + Business Media; 2007. xiv, 420-xiv, p.

4. Health AIo, Welfare. Comorbidity of mental disorders and physical conditions 2007. Canberra: AIHW, 2012.

5. Levenson JL, Hamer RM, Rossiter LF. Relation of psychopathology in general medical inpatients to use and cost of services. The American journal of psychiatry. 1990;147(11):1498-503. 
6. Saravay SM, Steinberg MD, Weinschel B, Pollack S, Alovis N. Psychological comorbidity and length of stay in the general hospital. The American journal of psychiatry. 1991;148(3):324-

7. Fulop G, Strain JJ, Fahs MC, Schmeidler J, Snyder S. A prospective study of the impact of psychiatric comorbidity on length of hospital stays of elderly medical-surgical inpatients. Psychosomatics. 1998;39(3):273-80.

8. Koopmans GT, Donker MC, Rutten FH. Length of hospital stay and health services use of medical inpatients with comorbid noncognitive mental disorders: a review of the literature. Gen Hosp Psychiatry. 2005;27(1):44-56.

9. Desan PH, Zimbrean PC, Weinstein AJ, Bozzo JE, Sledge WH. Proactive psychiatric consultation services reduce length of stay for admissions to an inpatient medical team. Psychosomatics. 2011;52(6):513-20.

10.Cassem NH, Hackett TP. Psychiatric consultation in a coronary care unit. Annals of internal medicine. 1971;75(1):9-14.

11.Cree RA, Bitsko RH, Robinson LR, Holbrook JR, Danielson ML, Smith C, et al. Health Care, Family, and Community Factors Associated with Mental, Behavioral, and Developmental Disorders and Poverty Among Children Aged 2-8 Years United States, 2016. MMWR Morb Mortal Wkly Rep . 2018 Dec 21;67(50):1377-1383.

12.Ercan ES, Polanczyk G, Akyol Ardic U, Yuce D, Karacetın G, Tufan AE, et al. The prevalence of childhood psychopathology in Turkey: a crosssectional multicenter nationwide study (EPICPAT-T). Nordic journal of psychiatry. 2019;73(2):132-40.

13. Shugart MA. Child psychiatry consultations to pediatric inpatients: A literature review. General hospital psychiatry. 1991;13(5):325-36.

14.De Hert, M, Correl Cu, Bobbes J, Bakmas M, Cohen D, Asai I, et al. Physical illness in patients with severe mental disorders. I. Prevalence, impact of medications and disparities in health care. World Psychiatry. 2011;10: 52-77.

15.Colpan M, Eray S, Vural P. Evaluation of Consultations Requested from the Departments of Child and Adolescent Psychiatry of Uludağ University Hospital within the Previous Year.The journal of current pediatrics. 2013; 11(3): 102-6.

16.Simsek EGE, Eyuboglu M, Eyuboglu D. Evaluation of Child and Adolescent Psychiatric Consultations in A University Hospital Osmangazi Journal of Medicine. 2019;41(3):24856.
17. Gokcen C, Celik YI. The Evaluation Of Child And Adolescent Psychiatry Consultations from other Inpatient Clinics in a Training Hospital. Sakarya Medical Journal. 2011;1(4):140-4.

18 Emiroglu N, Aras S, Yalin S, Dogan O, Akay A. Evaluation of the child and adolescent psychiatric inpatient consultations. Anatolian Journal of Psychiatry. 2009;10(3):217.

19.DikeC G, Arabacı LB, Uzunoğlu G. An Analysis Of The Consultations Requested In The Child And Adolescent Clinic Of A Regional Psychiatric Hospital. Acıbadem University Health Sciences Journal 2020;11(3):418-422.

20. Ertek IE, Ozturk HM. Assessment of psychiatric consultations in an educational-research hospital and accuracy rates of recognition of psychiatric diseases. Turkish J Clinical Psychiatry.2019;22(3):338-346.

21.Erdogan E, Delibas DH, Eskut N. Characteristics of Psychiatry Consultations Requested from the Neurology Clinic, Diagnostic Congruence Rates between Psychiatrists and Neurologists. Journal of Contemporary Medicine.10(2):168-75.

22. Yazici K, Tot S, Yazici A, Erdem P, Buturak V, Okyay Y, et al. Can Medical Inpatients in Need of Psychiatric Help Be Recognized? Turkish J Clinical Psychiatry. 2003;6(1):27-31.

23.Rezaki B, Oy B, Kuçukkomurcu S. Child Psychiatry Consultations in a State Hospital for Children. Turkish Journal of Child and Adolescent Mental Health. 1998;5(1):34-8.

24. Woodgate M, Elena Garralda M. Paediatric Liaison Work by Child and Adolescent Mental Health Services. Child and adolescent mental health. 2006;11(1):19-24.

25.Carter BD, Kronenberger WG, Baker J, Grimes LM, Crabtree VM, Smith C, et al. Inpatient pediatric consultation-liaison: A case-controlled study. Journal of Pediatric Psychology. 2003;28(6):423-32.

26. Muriel AC, Wolfe J, Block SD. Pediatric palliative care and child psychiatry: a model for enhancing practice and collaboration. Journal of palliative medicine. 2016;19(10):1032-8.

27. Walter HJ, Kackloudis G, Trudell EK, Vernacchio L, Bromberg J, DeMaso DR, et al. Enhancing pediatricians' behavioral health competencies through child psychiatry consultation and education. Clinical pediatrics. 2018;57(8):958-69.

28. Kilic B, Runa U, Ayla A. A Preliminary Evaluation of Consultation-Liaison Psychiatry Services For Children at A University Hospital: Lessons Learned to Enhance Efficacy. Yeni Symposium. 2007;45. 
29.Kandil S, Aksu H, Yontem T, Aktepe E. One year evaluation of child psychiatr consultations in a university hospital.Ibn-i Sina Journal of Medical Sciences. 2003;8:173-7.

30.Alpaslan AH, Koçak U, Çobanoğlu C, Görücü Y, editors. Evaluation of Child and Adolescent Psychiatry Consultations in a University Hospital. Yeni Symposium. 2015;53(3):10-15

31.Goker Z, Guney E, Gulser D, Uneri O. The Evaluation of the Psychiatric Consultations of Children and Adolescents Hospitalised in a Training and Research HospitaTurkish J Pediatr Dis. 2014; 1: 17-24

32.Dil LM, Vuijk PJ. Emergency Presentations to an Inner-city Psychiatric Service for Children and Adolescents. Child Care in Practice. 2012;18(3):255-69.

33.Lois TF. Perspectives on Female Adolescent Development and Psychopathology. Adolescent Psychiatry. 2011;1(2):146-51.

34.Natsuaki MN, Klimes-Dougan B, Ge X, Shirtcliff EA, Hastings PD, Zahn-Waxler C. Early pubertal maturation and internalizing problems in adolescence: sex differences in the role of cortisol reactivity to interpersonal stress. J Clin Child Adolesc Psychol. 2009;38(4):513-24.

35.Gozacanlar Ozkan O, Yalin Sapmaz S, Kandemir $\mathrm{H}$. Evaluation of Child Psychiatry Consultations of Stayed Patients at Pediatric Clinic. J Clin Psy. 2017;20:287-293

36.Tekkalaki B, Patil VY, Chate SS, Patil NM, Patil $S$, Sushruth V. Pediatric referrals to psychiatry in a Tertiary Care General Hospital: A descriptive study. Journal of Mental Health and Human Behaviour. 2017;22(1):40.

37. Shaw RJ, Pao M, Holland JE, DeMaso DR. Practice Patterns Revisited in Pediatric Psychosomatic Medicine. Psychosomatics. 2016;57(6):576-85. https://doi.org/10.1016/j.psym.2016.05.006.

38.Dilts SL, Jr., Mann N, Dilts JG. Accuracy of referring psychiatric diagnosis on a consultationliaison service. Psychosomatics. 2003;44(5):40711.

39.Sertoz OO, Doganavsargil GO, Noyan MA, Altıntoprak E, Elbi H. Accuracy rates of recognition of psychiatric disorders by nonpsychiatry specialists in a consultation-liaison service of a university hospital. Bulletin of Clinical Psychopharmacolog. 2008;18(4):288-295
40.Eser B, Batmaz S, Songur E, Yıldız M, Aslan EA. Analysis of the psychiatric consultations for inpatients and from the emergency room in a university hospital: A comparison with studies from Turkey. J Clin Psy. 2018;21:278-89.

41.Donker T, Griffiths KM, Cuijpers P, Christensen H. Psychoeducation for depression, anxiety and psychological distress: a meta-analysis. BMC medicine. 2009;7(1):79.

42.Rummel-Kluge C, Pitschel-Walz G, Bäuml J, Kissling W. Psychoeducation in schizophreniaresults of a survey of all psychiatric institutions in Germany, Austria, and Switzerland. Schizophrenia bulletin. 2006;32(4):765-75.

43. Dogangun B, Demirdogen ES. Consultation Liaison Psychiatry in Child and Adolescent in Treatment Options. J Child Psychiatry-Special Topics. 2016;2(2):95-100. 